\title{
Extrusion of screws with sensor functionality
}

\author{
Alexander Graf(1), Anna Guk ${ }^{1)}$, Peter Birnbaum ${ }^{1)}$ Verena Kräusel ${ }^{11}$, Andrey Afonin'2), Dirk Landgrebe ${ }^{1)}$, \\ Andreas Hirsch ${ }^{3)}$ \\ 1) Professorship for Forming and Joining, alexander.graf@mb.tu-chemnitz.de, \\ anna.guk@mb.tu-chemnitz.de, peter.birnbaum@mb.tu-chemnitz.de, \\ verena.kraeusel@mb.tu-chemnitz.de, uff@mb.tu-chemnitz.de, Chemnitz University of Technology, \\ Reichenhainer Straße 70, 09107 Chemnitz, Germany \\ 2)Institute of Engineering Technology and Natural Science, afonin@bsu.edu.ru, Belgorod National \\ Research University, Pobeda Street 85-14, 308015 Belgorod, Russia \\ 3) Professorship of Machine Tool Design and Forming Technology, wzm@mb.tu-chemnitz.de, \\ Chemnitz University of Technology, Reichenhainer Straße 70, 09107, Chemnitz, Germany
}

\section{Keywords}

Extrusion, FE Simulation, Piezoceramic Compound, Sensor Functionality, Structural Health Monitoring

\begin{abstract}
The use of components with embedded sensors is one of the most promising trends in civil engineering, plant construction and mechanical engineering. These components make it possible to both monitor the design state in real time and to recognize failures in parts and components early on. However, due to high product costs, their wider distribution is limited. Optimization of the component manufacturing technology can contribute to reducing the production costs.
\end{abstract}

Fasteners, such as screws and rivets, consisting of a polypropylene composite core filled with piezoceramic powder represent a potential approach. In the event of a sudden impact, this compound generates an electric pulse, and through machine learning the cause of the failure can be filtered out from the screw's normal state.

Simultaneous forming of aluminum and plastics opens up new opportunities to produce the abovementioned screws at a reasonable price. The authors' aim was to demonstrate feasibility through initial experiments and the generation of a simulation model.

\section{Introduction}

Lightweight design is the most essential method to improve the efficiency of vehicles and conserve resources. A special emphasis should be placed on material substitution. This design includes not only new higher-strength materials, but also lightweight metals and plastics. Although the latter withstand mechanical loads to a much lesser extent than metals do, it is possible to combine them with other materials and embed new functionalities.

The technologies to produce these components are based on the fundamental principles of roll cladding; an inseparable material bond is created by thermo-mechanical joining. The majority of the techniques investigated to date consider these compounds in sheet metal forming. In this field, the roll cladding procedure offers great potential for the manufacture of hybrid laminates with piezoceramic elements. The process consists of several steps, including the aluminum coil with structured surface and joining of the extruded piezo-film as well as the copper electrodes. Next, the finished laminate is polarized and characterized. The application-ready shape can be achieved by additional sheet forming processes such as deep drawing [1], [2]. The procedure for manufacturing functional elements according to Müller [3] provides another option: In this technique, microcavities are created in the aluminum sheet, which is joined with piezoceramic fibers, and the functional compound is subsequently deep-drawn. 
Bulk metal forming processes are particularly important for this task; however, researchers have not yet conclusively assessed their suitability. The work concerning these processes has mainly adapted the results of sheet metal processing to bulk forming. Schikorra has explored the possibility of increasing functional elements' strength and stiffness by extrusion of aluminum profiles with reinforcing and functional wires [4]. He paid special attention to the location of the wire inside the profile. Förster [5] has proposed two additional process variants: eccentric hydrostatic extrusion of Al-Mg composites and the production of structural components by forging. As a result, forging has demonstrated adequate formability for such compounds. Förster's approach was refined by Graf [6]. He verified a satisfactory agreement of the experimental results and the FE forging simulation - as well as of the Al-Mg and the aluminum-plastic composites. A way to produce smart components through incremental forming procedures without damaging the sensitive functional parts is shown in [7]; the researchers explored the influence of the axial fixture of a tube when integrating a ring part by means of rotary swaging. Only a few sources listed here comprise a numerical process illustration for a complete understanding of the process. Extrusion has not been considered in relation to composite production so far. Above all, the determination of the procedural limitations for the materials used have to be fundamentally investigated. In this publication, the authors focus on these open questions.

\section{Motivation}

In [1] and [2], flat material composites are formed into hybrid laminates with sensor functionalities by roll cladding and subsequent deep drawing. The authors have verified the scalability of this technique for large-series production. This raises the question of whether this would also work with bulk metal profiles and/or if it could be transferred to a typical mass product such as a screw. Brenneis [7] demonstrates that this principle works when swaging piezoceramic rings. Another approach consists of embedding piezoceramic particles into a thermoplastic matrix, see [1] and [2]. The thermoplastic matrix enables new manufacturing options for such composites. A process chain for the production of screws with sensor functions is illustrated in Figure 1. In the first step, the semi-finished product is joined with the aluminum cylinder, the piezo-compound and the copper electrode. In the second step, a semi-finished product for screw manufacturing is shaped by extrusion. After upsetting the head and thread production, the screw with sensor function is ready for use, e.g. in structural health monitoring. This paper focuses on the extrusion of the material compound.

semi-finished product

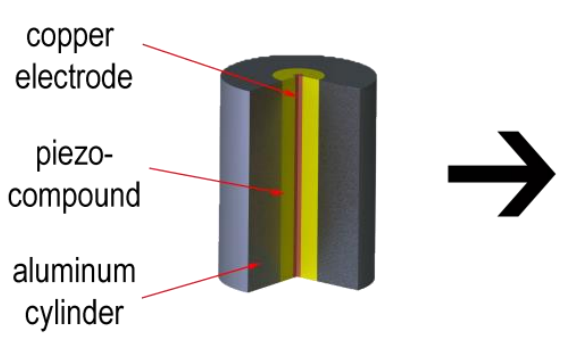

product after extrusion

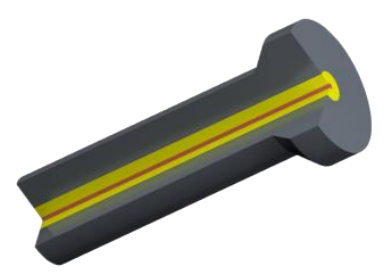

final product: hexagon head screw

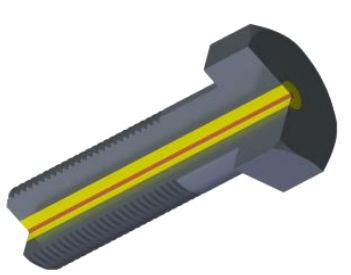

Figure 1: Structure and process chain of the screw with sensor functionality (shown as $270^{\circ}$ section)

\section{FE simulation}

\subsection{Model structure and parameters}

The authors created an FE model in DEFORM 2D to extrapolate the flow characteristics of the individual components during extrusion. The axial symmetry made it possible to model in 2D. The workpiece was assumed to be rigid plastic and the tool as ideally rigid. The researchers were able to store the mechanical workpiece properties as multi-linear flow curves determined in the experiment in the FE software (Figure 2). In this exploration, the copper electrode was not considered, based on the assumption that it is only slightly formed, and that the main focus of this analysis is on the interface between plastics and aluminum. The alloy AW 6082 in the T4 state was selected as screw production 
material. This alloy is frequently used in extrusion. The piezo-compound composition used corresponds to that in [1] and [2] (polypropylene (Moplen HP501H by LyondellBasell from Rotterdam/ the Netherlands) +70 wt. \% PZT +0.5 wt. \% CNT).

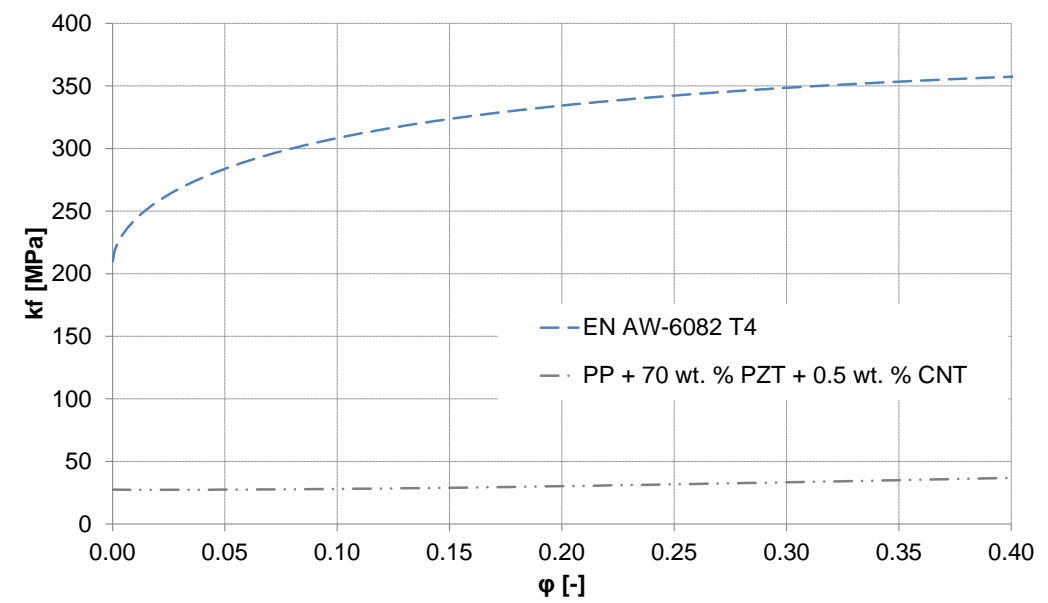

Figure 2: Yield curves of EN AW-6082 T4 and polypropylene at $70 w t . \% P Z T+0.5 w t . \%$ CNT

The geometry for modeling the workpiece was created in the pre-processor of the DEFORM software. The points were meshed by means of Lagrange tetrahedron elements. The researchers built up the workpiece geometry in the CAD system and exported it into DEFORM. The die taper angle was $15.5^{\circ}$. The initial model geometry is shown in Figure 3.

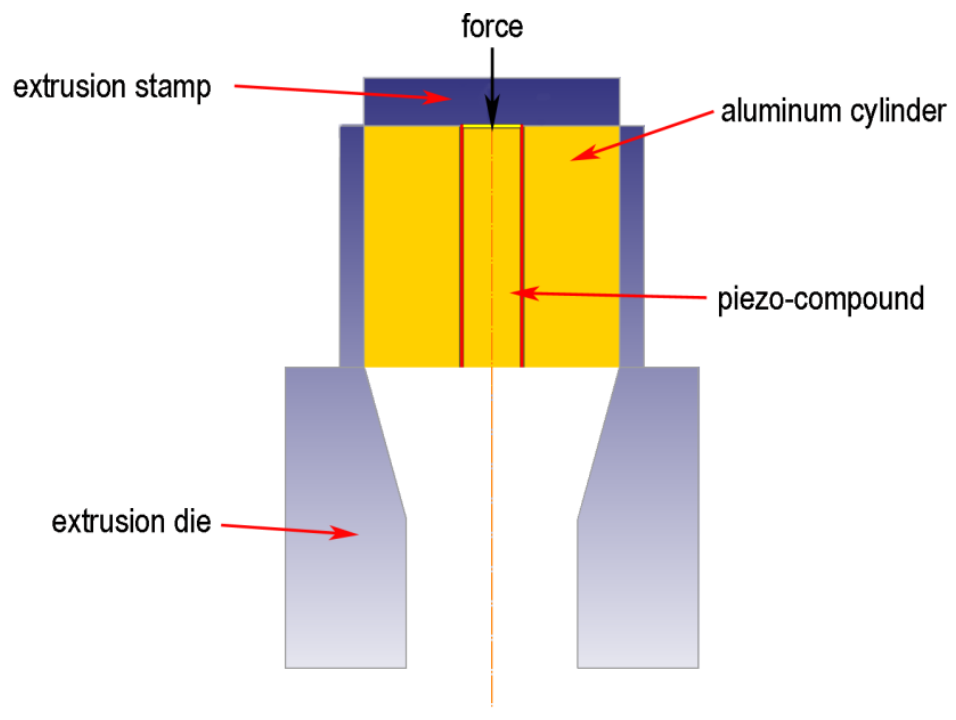

Figure 3: Initial geometry for the FE model

The initial and boundary conditions in the modeling were varied in the following ranges:

- Hole diameter for the piezo-compound: $1.5 \mathrm{~mm}-5 \mathrm{~mm}$

- Coefficient of friction between die and workpiece: $0.08-0.4$

Coefficient of friction between aluminum and plastic core: $0.08-1.0$

- Process temperature: $20^{\circ} \mathrm{C}-140{ }^{\circ} \mathrm{C}$

- Pressing speed: $1 \mathrm{~mm}-100 \mathrm{~mm} / \mathrm{s}$ 


\subsection{FE simulation results}

Polypropylene emerges from the hole due to the distinct mechanical properties of the screw corpus and the plastic core. The volume of the emitted material depends significantly on the difference in the mechanical properties of the materials used: The greater the difference, the more and softer the material flows out. The coefficient of friction between the aluminum and plastic material also affects the intensity of the streaming plastic: The higher the friction, the weaker the stream (Figure 4). The polypropylene deformation slightly decreases as a function of the increasing pressing speed (Figure 5). The plastic emerging from the hole results in a marked heating of the material as a result of friction (Figure 6). This effect contributes to the formation of a boundary between aluminum and the piezo-compound. As a result, it is possible to reduce the preheating temperature, thus saving energy.

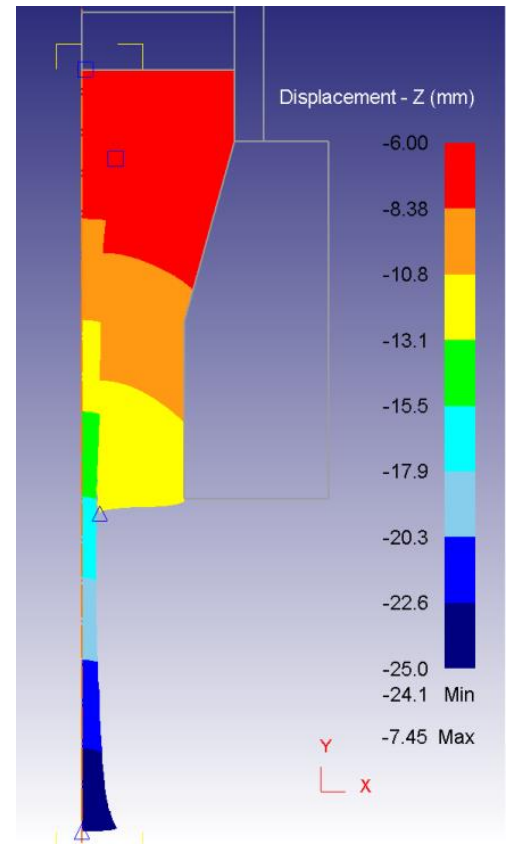

0.08

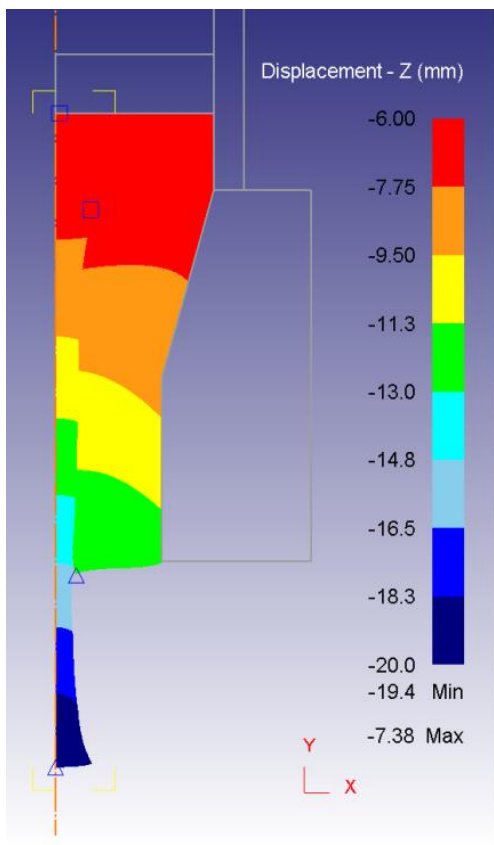

0.12

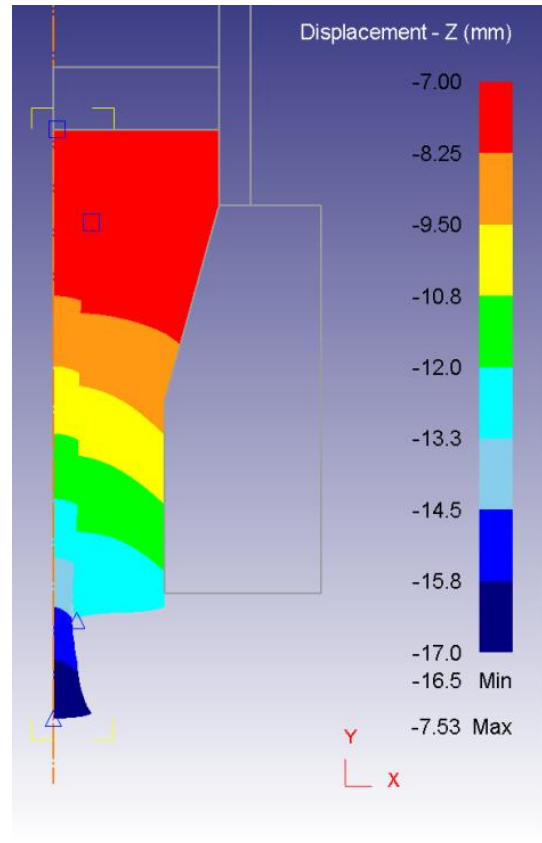

0.25

Figure 4: Axial displacement of the workpiece material as a function of the coefficient of friction between aluminum and polypropylene 


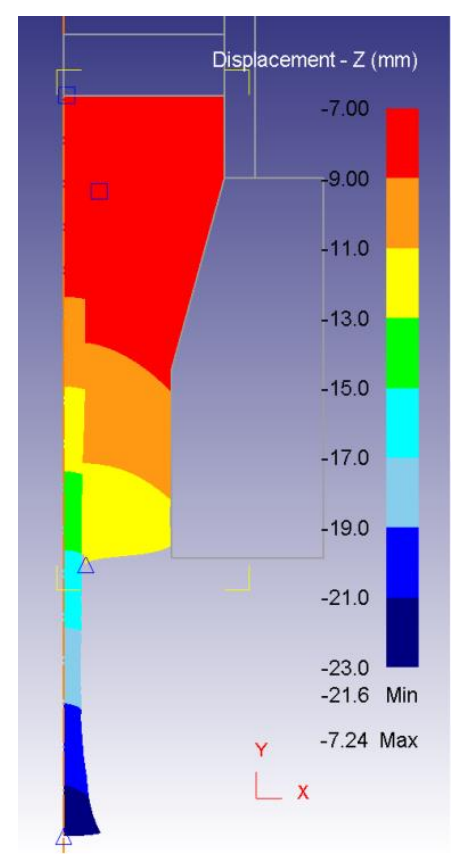

1

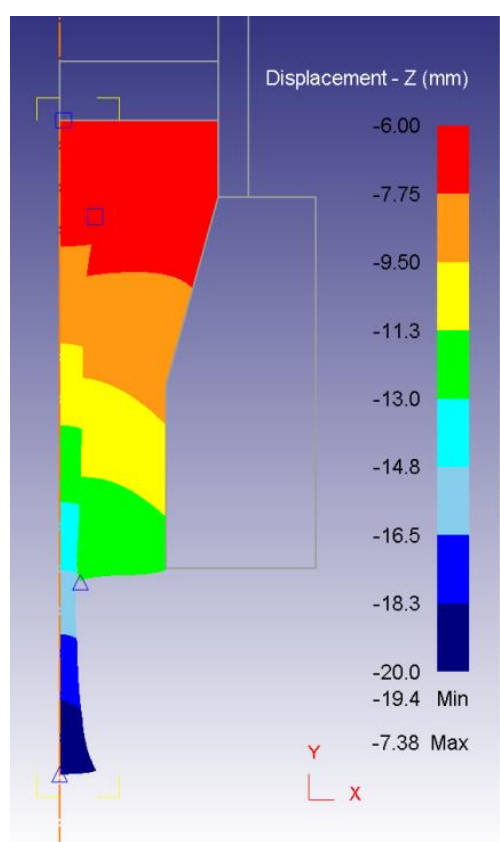

10

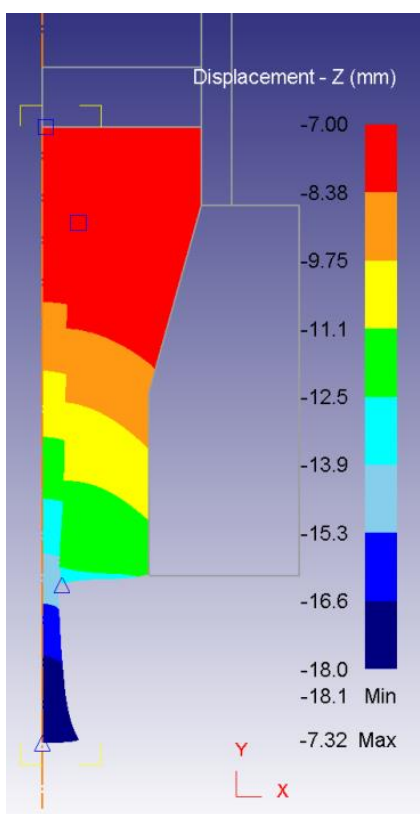

100

Figure 5: Material displacement of the workpiece in axial direction as a function of the pressing speed $[\mathrm{mm} / \mathrm{s}]$

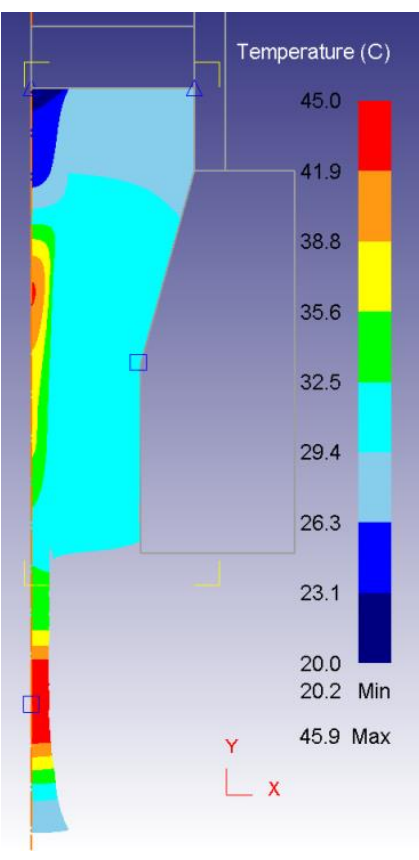

1

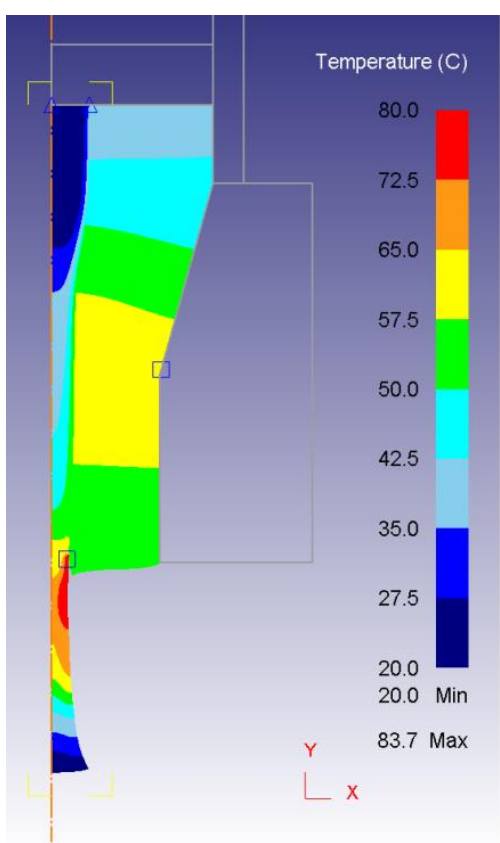

10

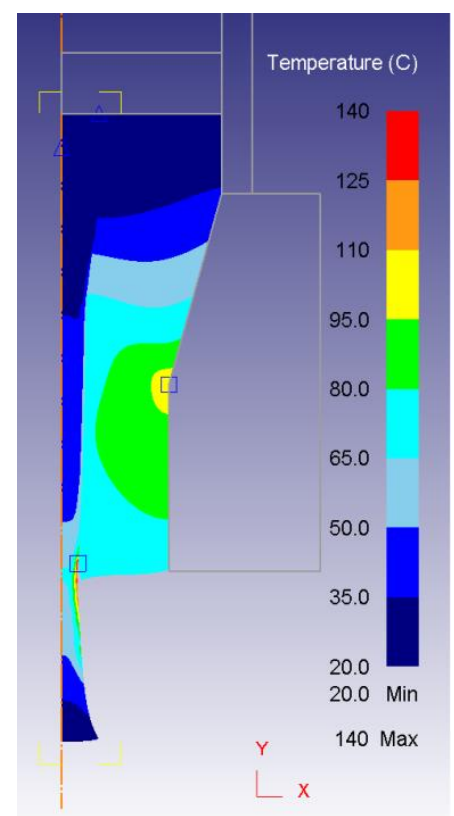

100

Figure 6: Workpiece heating as a function of the pressing speed $[\mathrm{mm} / \mathrm{s}]$

The outflow of the compound can be minimized by several measures; two variants are introduced in Figure 7: The introduction of shape elements, such as threads, is shown on the left. The situation is significantly improved compared to previous simulation results. The second variant is a numerical adaption of the boundary; cohesive elements between aluminum and plastics are modeled. Adhesive bonding between the materials is simulated by specifying a failure criterion. This can be transferred to several surface treatments depending on the application. This variant already significantly reduces the stream out of the plastics. 


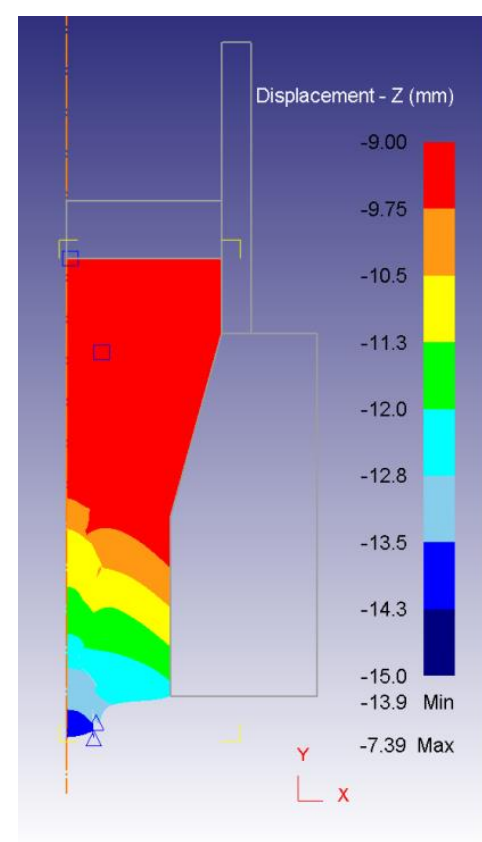

Thread in hole

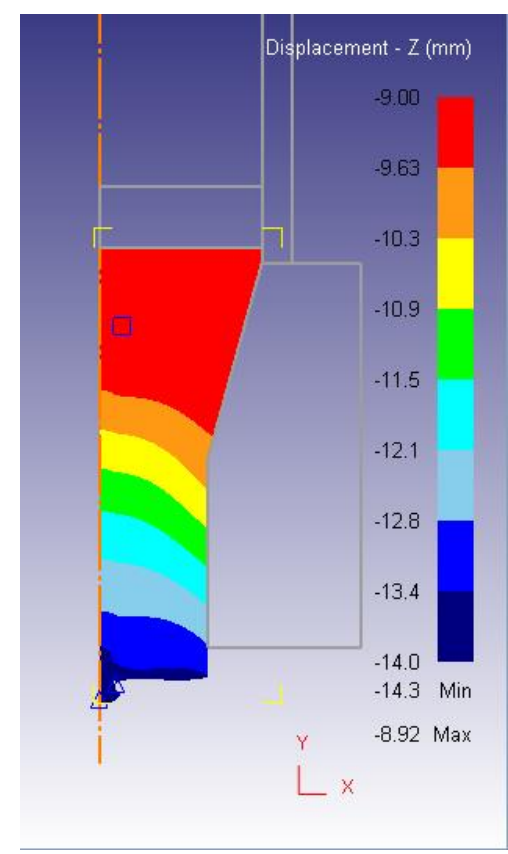

Cohesive zone between aluminum and polypropylene

Figure 7: Comparison of measures to avoid outflow of piezo-compound

\section{Extrusion experiments}

\subsection{Setup and parameters}

A die in an electric servo screw press by Dunkes was used for the extrusion test. The profile's initial diameter of $10.5 \mathrm{~mm}$ was reduced to $7.09 \mathrm{~mm}$ after extrusion, which corresponds to a tangential true strain of $\varphi_{t}=-0.39$. The researchers decided to design the hole for the piezo-compound insert as blind hole based on simulations and problems when handling the specimens. Furthermore, the following parameters were investigated:

- Hole of the piezo-compound: $1.8 \mathrm{~mm}$ and $2.4 \mathrm{~mm}$

- Pre-heating temperature: room temperature (RT) and $120^{\circ} \mathrm{C}$

- Electrode: without and with

Metallographic methods (cutting, grinding, polishing) and observation using an incident light microscope were employed to visualize the parameter effects on the transverse microsection.

\subsection{Experimental results}

The results in the form of transverse microsections are summarized in Figure 8 . In this analysis, the distinction between working with and without pre-heating is shown. For ongoing analysis, the image content was subdivided into sections of head, forming zone and shank. The macroscopic images in Figure 8 show that the dissipated thermal energy released during forming is insufficient to prevent these many small and large pores. In contrast, it has been possible to significantly reduce the pores in the preheated specimen. Fehler! Verweisquelle konnte nicht gefunden werden. compares enlarged sections of the individual zones to allow a much more detailed analysis of these effects. The magnification provides a better understanding of the first assumption regarding the pores. As we can see in the experiment without pre-heating, there is no longer an adhesive bond at the boundary between the piezo-compound and aluminum (Fehler! Verweisquelle konnte nicht gefunden werden. a) due to delamination. By contrast, pre-heating (Fehler! Verweisquelle konnte nicht gefunden werden. b) of the specimens clearly enhances bonding. 
Technologies for Lightweight Structures 1(1) (2017), pp. 24-32

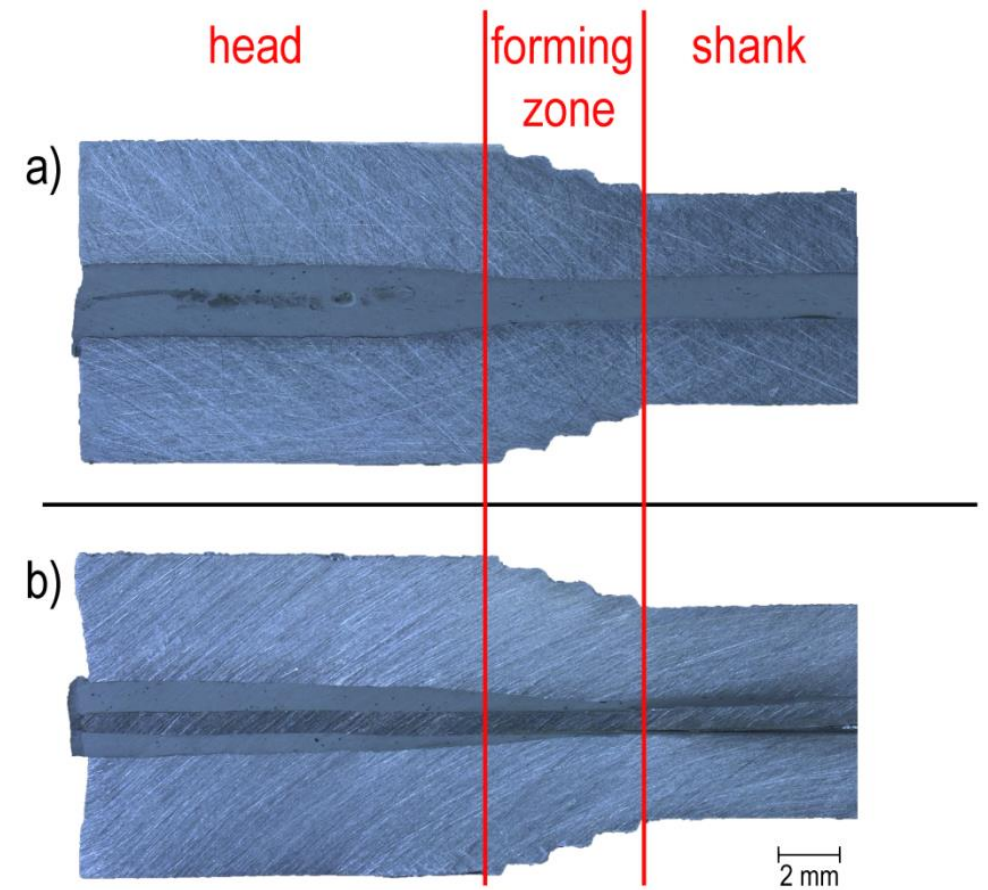

Figure 8: Cross-sections of extruded specimens: a) without and b) with pre-heating

Table 1 Detailed view of metallographic sections of the formed screw with sensor functionality a) without and b) with pre-heating

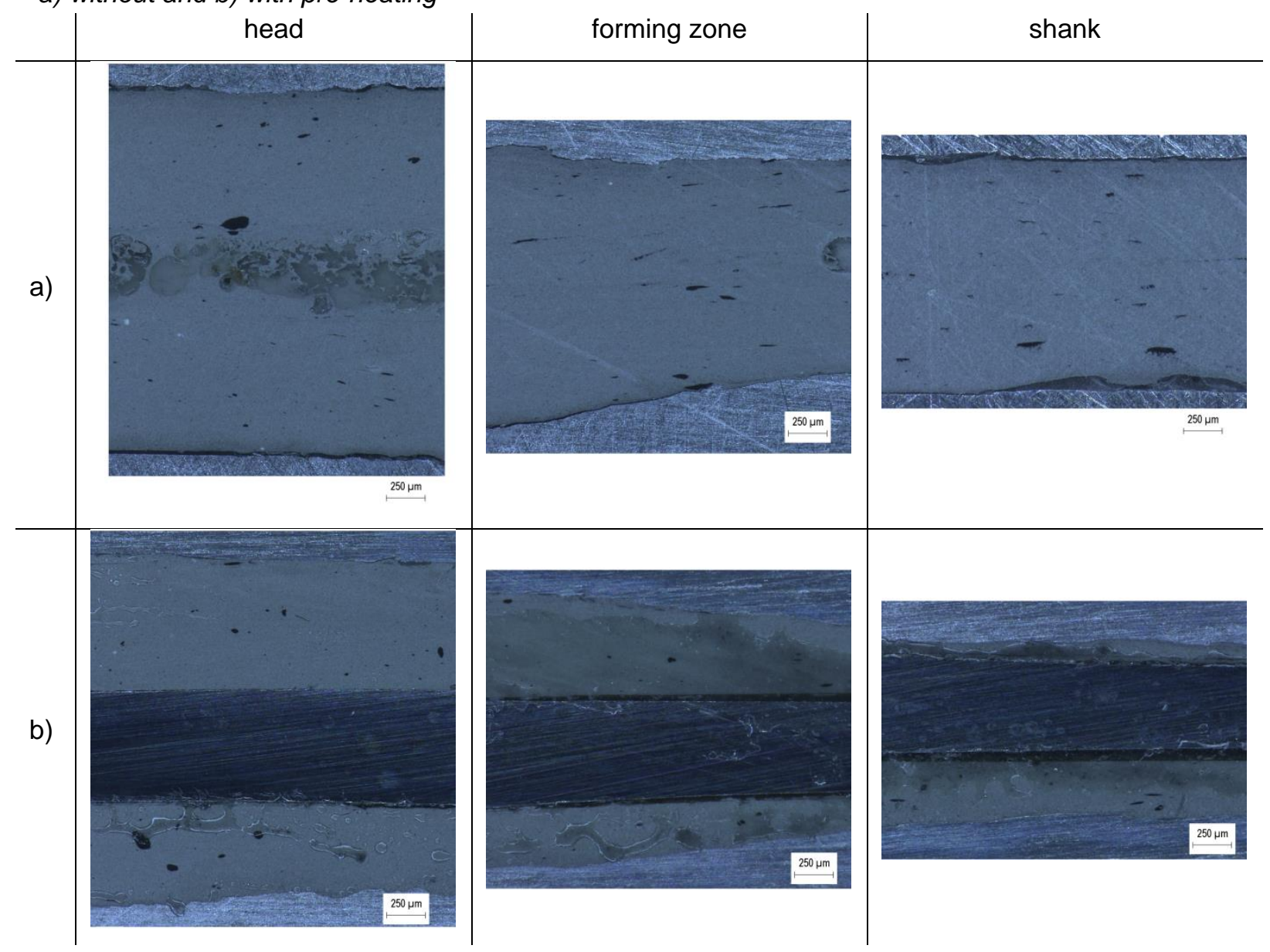


The transverse microsection diameters of the individual zones and components were measured with a digital microscope. Assuming that the head remains unformed, these diameters were defined as initial diameter $D_{0}$, and the diameters in the shank zone after forming as $D_{u}$. Based on the equation

$$
\varphi_{t}=\ln \left(\frac{D_{U}}{D_{0}}\right)
$$

the authors were able to calculate the tangential true strain. The results are summarized in Figure 9 and thus distinguish between the piezo-compound parameter $D$ and that of the pre-heating temperature. The authors succeeded only in quantifying the electrode forming ratio in one experiment. The parameters explored obviously do not significantly affect the forming of the aluminum cylinder, but strongly influence the piezo-compound's forming ratio. The maximum forming ratio is achieved by forming with the minimal hole $D$. A significant increase in the forming ratio was achieved in the experiment with pre-heating. The larger tangential forming ratio causes the plastic material to flow more easily into the microstructure of the aluminum cylinder and also provides an explanation for the boundary without delamination, as shown in Figure 8. When the electrode's forming ratio was assessed, it turned out to be almost without deformation. From this phenomenon we can conclude that the electrode in the soft plastic matrix also flows and that no forces are transformed which could have resulted in plastic deformation.

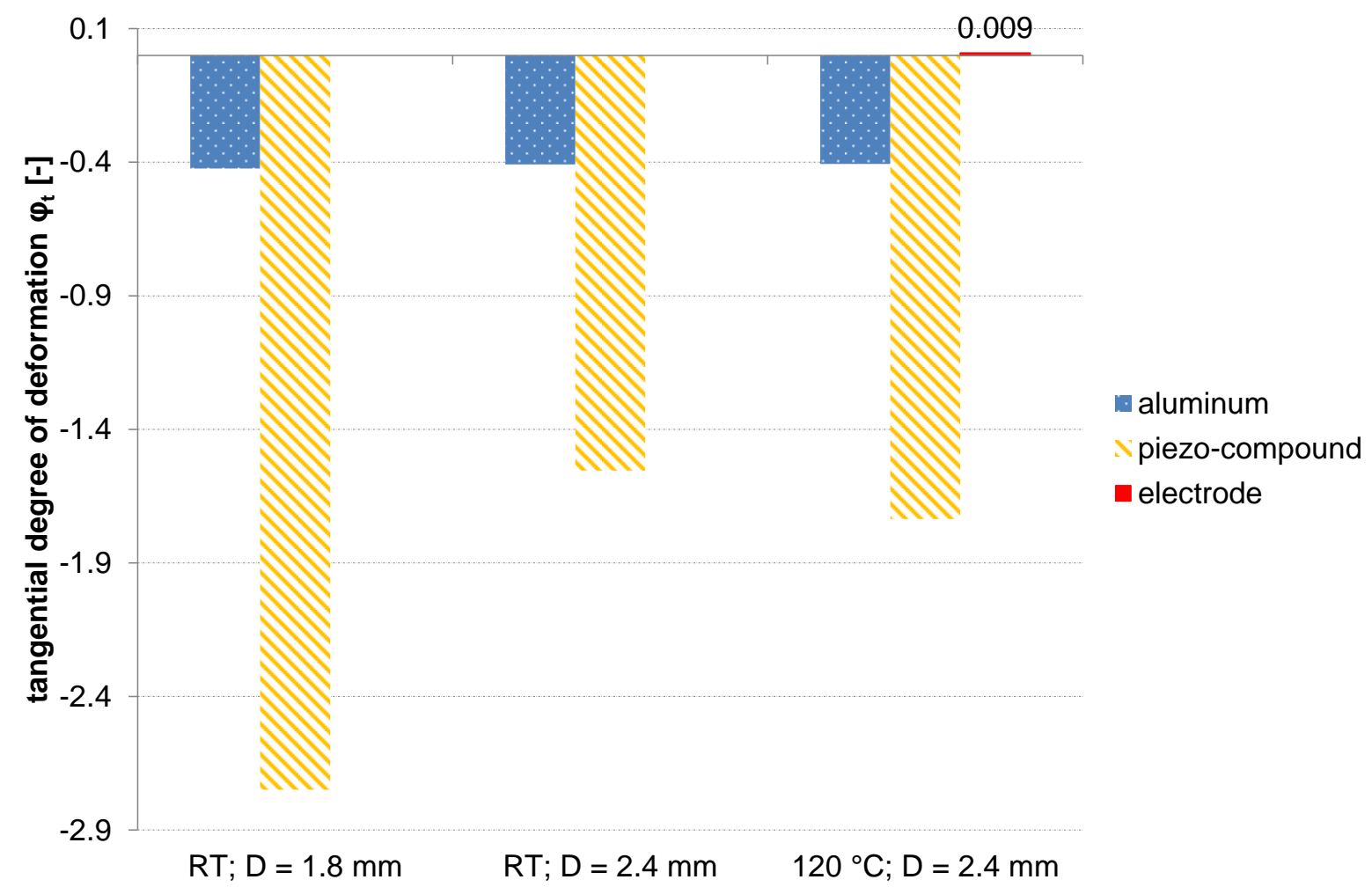

Figure 9: Measured tangential forming ratios in comparison as a function of pre-heating and hole diameter $D$

\section{Summary}

The extrusion of screws with sensor functionalities is challenged by several technological problems in comparison with conventional screw manufacturing. One reason for this is that aluminum alloys and plastics differ greatly in their mechanical properties.

Basic information about the extrusion of screws with sensor functionalities could be obtained with an FE model. The calculations showed that the deformation in plastics is transformed into heat as a function of the stamp speed. This phenomenon is very important for practical tests. By using shape elements in the hole, the outflow of plastic can be minimized, and the modeling of cohesive elements is a measure to immediately affect adhesive bonding between the metal and the plastic. 
As the practical experiments show, it is necessary to pre-heat the specimens to $120^{\circ} \mathrm{C}$ to minimize the formation of pores in the plastic material and to guarantee adequate boundary adhesion between the metal and the plastic. Having evaluated the tangential forming ratio of the individual components, better flow in the tangential piezo-compound direction could be confirmed at higher temperatures. Future experiments will determine the influence of the forming rate with the intention of reducing the pre-heating temperature further through higher heat dissipation during forming. The signal intensity as a function of the manufacturing conditions also has to be determined.

\section{Acknowledgements}

We gratefully acknowledge the cooperation of our project partners and the financial support of the German Research Foundation (DFG) within the Federal Cluster of Excellence EXC 1075 "MERGE".

\section{References}

[1] Ullmann, F.; Decker, R.; Graf, A.; Kräusel, V.; Heinrich, M.; Hardt, W.; Kroll, L.; Landgrebe, D.: Continuous Manufacturing of Piezoceramic Hybrid Laminates for Functionalised Structural Components. Technologies for Lightweight Structures, 1 (2017), pp. 1-13. doi:10.21935/tls.v1i1.64

[2] Kräusel, V.; Graf, A.; Heinrich, M.; Decker, R.; Caspar, M.; Kroll, L.; Hardt, W.; Göschel, A.: Development of hybrid assembled composites with sensory function. CIRP Annals Manufacturing Technology, 64 (2015) 1, pp. 25-28. doi:10.1016/j.cirp.2015.04.054

[3] Müller, M.; Müller, B.; Hensel, S.; Nestler, M.; Jahn, S.F.; Wittstock, V.; Schubert, A.; Drossel W.G.: Structural Integration of PZT Fibres in Deep Drawn Sheet Metal for Material-integrated Sensing and Actuation. 2nd International Conference on System-Integrated Intelligence: Challenges for Product and Production and Production Engineering. Procedia Technology, 15, 2014, pp. 659-668.

[4] Schikorra, M.; Tekkaya, A. E.; Kleiner, M.: Experimental Investigation of embedding High Strength reinforcements in Extrusion Profiles. CIRP Annals - Manufacturing Technology, 57 (2008), pp. 313-316. doi:10.1016/j.cirp.2008.03.024

[5] Förster, W.; Binotsch, C.; Awiszus, B.; Lehmann, T.; Müller, J.; Kirchbach, C.; Stockmann, M.; Ihlemann, J.: Forging of Eccentric Co-Extruded Al-Mg Compounds and Analysis of the Interface Strength. Materials Science and Engineering 118 (2016), pp. 1-10,

[6] Graf, M.; Härtel, S.; Feuerhack, A.; Förster, W.; Awiszus, B.: Schmieden von hybriden MetallKunststoff- und Metall-Metall-Verbunden. In: 23. Sächsischen Fachtagung Umformtechnik (SFU), 2016, pp. 204-213.

[7] Brenneis, M.; Groche, P.: Smart Components through Rotary Swaging. Key Engineering Materials, Vols. 504-506, pp. 723-728. 